CASE REPORT

\title{
Benign mixed tumour of the skin with extensive ossification and marrow formation: a case report
}

\author{
R Awasthi, D Harmse, D Courtney, C B A Lyons
}

J Clin Pathol 2004;57:1329-1330. doi: 10.1136/jcp.2004.020172

Benign mixed tumour of the skin (chondroid syringoma) is an uncommon skin adnexal tumour, usually presenting as a slow growing solitary painless nodule. The morphological appearances are similar to those of a pleomorphic adenoma of the salivary gland. Hair matrix and sebaceous differentiation can be seen in some lesions. Focal ossification is a rare finding. This report presents a case of a similar tumour arising in the cheek of a 43 year old white man, showing extensive ossification. Clinical, radiological, and pathological correlation and diagnosis proved to be difficult preoperatively. Only two cases of a benign mixed tumour with pronounced ossification have been reported so far, both in Japanese patients. This is the first reported case seen in a white man. Awareness of these lesions will avoid potential diagnostic piffalls.

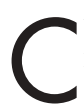
utaneous benign mixed tumour (chondroid syringoma) is an uncommon adnexal tumour usually presenting as a slow growing, painless nodule in the head and neck region-particularly the nose, cheek, and upper lip-of middle aged and elderly patients; malignant transformation is rare. Its histological appearances are similar to those of a pleomorphic adenoma of the salivary gland. Occasional tumours show pilosebaceous differentiation or focal calcification, but ossification is rare.

To the best of our knowledge, only two cases of cutaneous benign mixed tumour with pronounced ossification have been reported in the medical literature, both in Japanese patients. ${ }^{12}$ We present another case, arising in the cheek of a 43 year old white man.

\section{CASE REPORT}

A 43 year old man presented with a five year history of a slow growing lump on the left side of the face. On examination, the lesion was $5 \mathrm{~cm}$ in diameter, firm, non-tender, and freely mobile. There was no associated lymphadenopathy. A provisional diagnosis of pleomorphic adenoma of a minor salivary gland was made. A fine needle aspirate of the lesion was moderately cellular and contained cohesive groups of epithelial cells with plump hyperchromatic nuclei and moderate amounts of cytoplasm; some cells showed keratinisation. The features were suggestive of a neoplastic tumour. Although accurate typing was difficult, the appearances supported the clinical diagnosis of a pleomorphic adenoma. A subsequent needle core biopsy was suggestive of pleomorphic adenoma with focal squamous differentiation.

A magnetic resonance imaging scan showed a $4 \mathrm{~cm}$ diameter nodule in the left cheek, which appeared to be well defined and composed of two distinct areas: in one area there was a rounded homogeneous nodule typical of a benign tumour; in the other, there were branching spiculate structures admixed with what appeared to be fat. These radiological appearances were confusing because the presence of irregular spiculate structures extending into fat raised the possibility of malignancy. In addition, no connection of the tumour to the salivary gland could be demonstrated.

The histopathology of the needle core biopsy could not be reconciled with the radiological findings. A decision was therefore made at the multidisciplinary meeting to excise the lesion completely, but not to compromise any vital structures (for example, nerves), before a definitive diagnosis was made.

Histological examination of the excision specimen showed a well circumscribed tumour within the deep dermis and subcutaneous fat, with no obvious involvement of the superficial skin. The tumour was composed predominantly of bone including cellular marrow fat with erythroid elements. In other areas the features were typical of a benign mixed tumour (fig 1). Focally, there were numerous hair follicle-like structures and keratocysts, indicating pilar differentiation, and in some areas pilomatricoma-like features (fig 2), with typical basaloid and shadow cells, were prominent. Occasional groups of apocrine glands were also present. No cellular atypia was seen, and only occasional mitoses were found. The low proliferative activity of the tumour was confirmed with by MIB-1 immunochemistry.

\section{DISCUSSION}

Typically, benign mixed tumours of the skin contain an intimate admixture of epithelial-myoepithelial structures within a chondromyxoid and fibrous stroma. Occasionally, differentiation towards various skin adnexal structures (including hair matrix, hair follicle, apocrine, and sebaceous glands) is encountered, suggesting origin from a

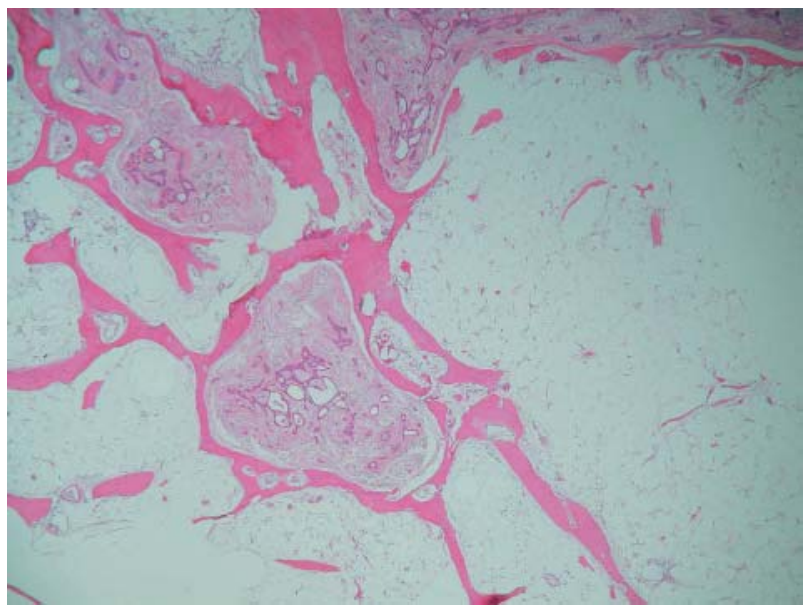

Figure 1 An area of extensive ossification and fatty marrow closely intermixed with a typical area of benign mixed tumour. 


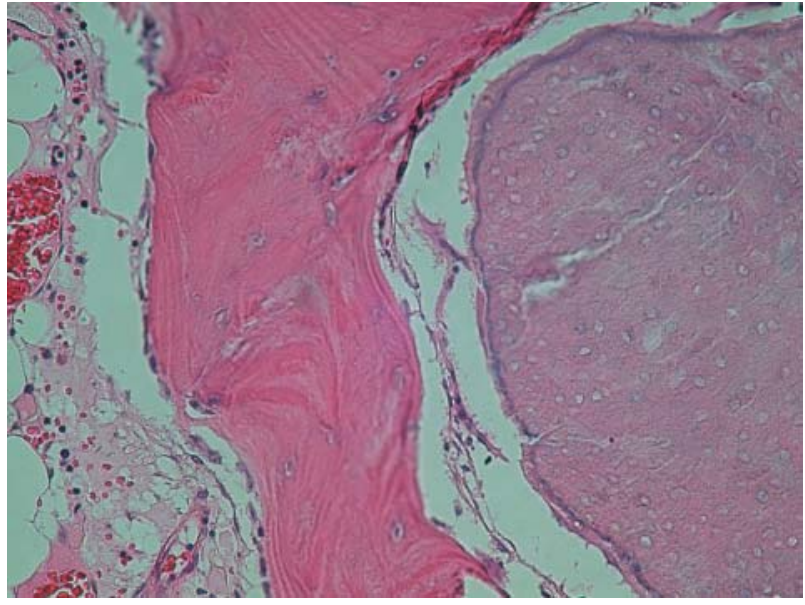

Figure 2 A bony trabecula in close apposition to a pilomatricoma-like area.

folliculosebaceous-apocrine unit. $^{3}{ }^{4}$ The generally accepted view is that there are apocrine and eccrine variants. Follicular differentiation is more commonly seen than sebaceous. ${ }^{4}$ Ossification is a rare feature and when present is usually focal and scant. ${ }^{1}$ Tsoitis et al reported a case of benign mixed tumour arising in the skin of the temple and showing focal ossification and pilosebaceous differentiation. ${ }^{5}$

Extensive ossification in benign mixed tumours at any anatomical site is exceedingly rare, and so far, only two such tumours have been reported in the skin, both in Japanese patients. ${ }^{12}$ Akasaka et al suggested that ossification in these tumours indicates development from pluripotential stem cells. ${ }^{2}$

Apart from the rare occurrence of ossification in cutaneous mixed tumours of the skin, secondary (metaplastic) ossification may be seen in a variety of other skin lesions, including naevi (particularly on the face (osteonaevus of Nanta)), basal cell carcinomas, up to $20 \%$ of pilomatricomas, and less commonly in trichoepitheliomas, haemangiomas, pyogenic granulomas, schwannomas, lipomas, organoid naevi, epidermal and dermoid cysts, dermatofibromas, desmoplastic melanomas, and some cutaneous metastases. ${ }^{6}$

"Extensive ossification in benign mixed tumours at any anatomical site is exceedingly rare, and so far, only two such tumours have been reported in the skin, both in Japanese patients"

\section{Take home messages}

- Benign mixed tumours of the skin occasionally show differentiation towards other skin adnexal structures

- Focal ossification is sometimes seen but extensive ossification is a rare feature

- Such appearances can pose diagnostic difficulty and clinical, radiological, and pathological correlation is essential

In conclusion, we present a case of benign mixed tumour of the skin with extensive ossification. This case, to the best of our knowledge is the first to be reported in a white patient. The presence of extensive ossification with areas of cellular marrow fat caused diagnostic difficulty, particularly on magnetic resonance imaging, and the pathological appearances of a needle core biopsy could not be reconciled with radiology. Awareness of this phenomenon in benign mixed tumour of the skin could avoid future diagnostic pitfalls.

\section{Authors' affiliations}

R Awasthi, D Harmse, C B A Lyons, Department of Histopathology, Derriford Hospital, Derriford Road, Plymouth PL6 8DH, UK

D Courtney, Department of Maxillofacial Surgery, Derriford Hospital

Correspondence to: Dr R Awasthi, Department of Histopathology, Derriford Hospital, Derriford Road, Plymouth PL6 8DH, UK; rachnaawasthi@hotmail.com

Accepted for publication 23 June 2004

\section{REFERENCES}

1 Shimizu S, Han-Yaku H, Fukushima S, et al. Immunohistochemical study of mixed tumour of the skin with marked ossification. Dermatology 1996; 193:255-7.

2 Akasaka T, Onodera H, Matsuta M. Cutaneous mixed tumour containing ossification, hair matrix, and sebaceous ductal differentiation. J Dermatol 1997:24:125-31.

3 Yamamoto O, Yasuda H. An immunohistochemical study of the apocrine type of cutaneous mixed tumors with special reference to their follicular and sebaceous differentiation. J Cutan Pathol 1999;26:232-41.

4 Requena L, Sanchez Yus E, Santa Cruz DJ. Apocrine type of cutaneous mixed tumour with follicular and sebaceous differentiation. Am J Dermatopathol 1992; 14:186-94.

5 Tsoitis G, Brisou B, Destombes P. Mummified cutaneous mixed tumour. Arch Dermatol 1975;111:194-6.

6 Weedon D. Cutaneous deposits. In: Houston MJ, ed. Skin pathology, 2nd ed. Edinburgh: Churchill Livingston, an imprint of Elsevier Science Ltd, 2003:427. 